\title{
Spherically-symmetric Accretion onto a Black Hole at the Center of a Young Stellar Cluster
}

\author{
Sergiy Silich, Guillermo Tenorio-Tagle \\ and \\ Filiberto Hueyotl-Zahuantitla \\ Instituto Nacional de Astrofísica Optica y Electrónica, AP 51, 72000 Puebla, México; \\ silich@inaoep.mx
}

\begin{abstract}
Here we present a self-consistent, bimodal stationary solution for spherically symmetric flows driven by young massive stellar clusters with a central supermassive black hole $(\mathrm{SMBH})$. We demonstrate that the hydrodynamic regime of the flow depends on the location of the cluster in the 3D (star cluster mechanical luminosity - BH mass - star cluster radius) parameter space. We show that a threshold mechanical luminosity $\left(L_{\text {crit }}\right)$ separates clusters which evolve in the $\mathrm{BH}$ dominated regime frome those whose internal structure is strongly affected by the radiative cooling. In the first case (below the threshold energy) gravity of the $\mathrm{BH}$ separates the flow into two distinct zones: the inner accretion zone and the outer zone where the star cluster wind is formed. In the second case (above the critical luminosity), catastrophic cooling sets in inside the cluster. In this case the injected plasma becomes thermally unstable that inhibits a complete stationary solution.

We compared the calculated accretion rates and the $\mathrm{BH}$ luminosities with those predicted by the classic Bondi accretion theory and found that Bondi's theory is in good agreement with our results in the case of low mass clusters. However, it substantially underestimates the accretion rates and $\mathrm{BH}$ luminosities if the star cluster mechanical luminosity, $L_{S C} \geq 0.1 L_{\text {crit }}$.
\end{abstract}

Subject headings: accretion — galaxies: active — galaxies: starburst — galaxies: star clusters — hydrodynamics

\section{Introduction}

Intensive studies of active galactic nuclei (AGNs) in the optical, infrared (IR) and Xray regimes during the last decade, have unveiled the presence of massive starbursts around 
the central supermassive black hole $(\mathrm{BH})$ in a number of Seyfert galaxies. For example, Rodríguez Espinosa et al. (1987) found that the 25, 60 and $100 \mu \mathrm{m}$ fluxes of classical optically selected Seyfert galaxies are not correlated with their ultraviolet (UV) and optical continuum and that far-IR colors of the selected galaxies are indistinguishable from those of starburst galaxies. They suggested then that high far-IR luminosities associated with many Seyfert galaxies indicate an intrinsic link between the circumnuclear star formation and the AGN activity. Baum et al. (1993) revealed a kiloparsec-scale, diffuse radio emission in 12 of the 13 observed Seyfert galaxies. They found that the intensity of the diffuse radio emission correlates with the far-IR luminosity of the host galaxy, suggesting that this emission is generated in the galactic superwind plasma driven by the nuclear starburst along the minor axis of the galaxy, and claimed that circumnuclear starbursts and starburst driven outflows may be intrinsic to many Seyferts although their relative strengths may vary from galaxy to galaxy.

Levenson et al. (2001) presented X-ray imaging and spectroscopy of a sample of 12 Seyfert 2 galaxies and concluded that in order to fit the observed X-ray spectra it is required to combine the power-law Seyfert component with a thermal starburst emission. JiménezBailón et al. (2005) presented XMM-Newton and Chandra observations of the Seyfert 2 galaxy NGC 1808. They found the hard X-ray emission associated with an unresolved nuclear sources whereas the soft emission is dominated by a thermal component associated with an extended starburst.

Terlevich et al. (1990) suggested to use the stellar CaII triplet absorption feature in the IR continuum as a direct indicator on the presence of young unresolved stellar population in the nuclear regions of Seyfert galaxies. Heckman et al. (1997) and González Delgado et al. (1998) found absorption line features associated with photospheres of O and B stars and their stellar winds in the ultraviolet and optical spectra of four Seyfert 2 galaxies: Mrk 477, NGC 7130, NGC 5135 and IC 3639 and thus presented direct evidence for the existence of nuclear starbursts in these galaxies. They found that the size of the nuclear starbursts in these galaxies ranges from several tens to a few hundred parsecs. The co-existence of W-R features in the optical and Ca II triplet in the near-IR part of the spectra, implies either a continuous star formation during more than $\sim 10$ Myr or two stellar generations with ages about 5-6 Myr and 10-20 Myr, respectively. Such starbursts are likely to drive the highvelocity outflows detected in the above and in Seyfert 2/starburst ultra luminous infrared galaxies (González Delgado et al. 1998; Rupke et al. 2005).

On the other hand, compact, bright stellar clusters or nuclear star clusters, were found in the centers of $\sim 75 \%$ of local spirals and Virgo dwarf elliptical galaxies (Böker et al. 2002; Côté et al. 2006). Their radii (a few parsecs) are similar to those of globular clusters, however 
they are 1 - 2 orders of magnitude brighter, more massive and may have complicated star formation histories with several episodes of star formation (Walcher et al. 2006). Ferrarese et al. (2006) claimed that massive galaxies $\left(\mathrm{M}_{\text {gal }}>10^{10} \mathrm{M}_{\odot}\right)$ host supermassive BHs whereas less massive galaxies host only nuclear clusters. However Seth et al. (2007) presented evidences on the presence of super massive black holes in $\sim 25 \%$ of galaxies which host nuclear star clusters. More than half of these galaxies $(\sim 15 \%)$ present a mixed AGNstarburst optical spectra and are classified as composite.

Shlosman \& Begelman (1989), Goodman (2003), Collin \& Zahn (1999), Tan \& Blackman (2005) have shown that accretion disks are gravitationally unstable outside of $r \sim 10^{-2} \mathrm{pc}$ and must fragment into self-gravitating clumps that eventually form stars. It was suggested that star formation reduces the rate of accretion and thus the luminosity of the central supermassive black hole (BH) by removing mass from the accretion flows and also due to radiative heating of the accretion discs. However non of these models took into consideration the negative feedback provided by the mechanical energy of the central starburst on the accretion flow.

Thus circumnuclear star formation occurs at different space scales around the SMBH in many AGN galaxies. Here we note that the mechanical power of young nuclear starbursts might prevent through the cluster winds the accretion of interstellar matter from the bulges and disks of their host galaxies onto the central BHs. In such cases the BHs are fed with the matter injected by numerous stellar winds and SNe explosions that result from the multiple evolving sources. This implies that nuclear starbursts must strongly affect and perhaps even control the power of the central BH. In fact, it may be the dominant factor to be consider in order to understand the physics and relative contributions of the $\mathrm{BH}$ and starburst activity to the energy budget of the composite AGN/starburst galaxies. Lípari \& Terlevich (2006) have incorporated different ingredients of this physics into their evolutionary unification scenario which seems to be able to explain many properties of AGNs and QSOs.

The classic spherically-symmetric accretion model (Bondi, 1952; Frank et al. 2002) should then be modified if one is to apply it to the case of a massive $\mathrm{BH}$ at the center of a young stellar cluster. First, it should take into consideration the energy and mass supplied by massive stars within the star cluster volume. Second, it should account for radiative losses of energy from the hot thermal plasma. Third, the models should incorporate initial and boundary conditions as described by the star cluster wind theory (Chevalier \& Clegg, 1985; Canto et al. 2000; Silich et al. 2004; Tenorio-Tagle et al. 2007).

Here we present a self-consistent semi-analytic theory of stationary spherically-symmetric flows driven by the thermalization of the kinetic energy supplied by massive stars inside massive stellar clusters which includes the outflow of the injected matter and its accretion onto 
a central massive BH. The solutions account for proper initial and boundary conditions for a variety of stellar clusters and black holes and the impact of strong radiative cooling on the dynamics of the thermalized injected matter.

The paper is organized as follows. In section 2 we formulate our model and discuss the input physics and major simplifications. In section 3 we present a set of major equations. Boundary conditions and the selection of the proper solution from a family of integral curves are discussed in section 4 . In section 5 we discuss the impact that a central BH provides on the star cluster driven flows. We discuss two hydrodynamic regimes separated in the star cluster mass vs star cluster radius diagram by a threshold line: the BH dominated regime below the threshold line and the radiative cooling dominated regime above the threshold line. In section 6 we use our model to calculate the accretion rates and $\mathrm{BH}$ accretion luminosities and compare them to those, predicted in the classical Bondi accretion theory. Section 7 summarizes our results and gives our conclusions.

\section{The model}

Following Chevalier \& Clegg (1985) we assume that massive stars are homogeneously distributed inside a spherical volume of radius $R_{S C}$ and that the mechanical energy deposited through stellar winds and supernovae, $L_{S C}$, is thermalized via random collisions of the gaseous streams from neighboring sources. This results into a high temperature and a high thermal pressure that leads to a fast outflow of the injected matter, while composing a stationary star cluster wind.

In presence of a massive, central black hole, a fraction of the deposited matter is to remain bound inside the cluster to eventually fall onto the center. This implies that in presence of a $\mathrm{BH}$ the stagnation point, the point where the expansion velocity, $u_{w}=0 \mathrm{~km}$

$\mathrm{s}^{-1}$, is not at the star cluster center, as it happens if the cluster does not contain a BH and evolves in the quasi-adiabatic regime (see below), but instead is at a distance, $R_{s t}$, from the star cluster center. The position of the stagnation point thus becomes an important issue that defines both the upper limit for the accretion rate onto the central $\mathrm{BH}$, and the amount of matter that the star cluster returns, in the form of a wind, to the interstellar medium.

As it was mentioned by Nulsen \& Fabian (2000), the dissipation of angular momentum is less of a problem for the hot plasma. Thus we restrict ourselves to spherically-symmetric solutions, despite realizing that some fraction of the injected material that remains bound inside the star cluster and that falls onto the center, eventually forms an accretion disk associated with the central black hole. We assume that this occurs at a sufficiently small 
radii and neglect in this paper all effects associated with the redistribution of the residual angular momentum.

We do not consider the relativistic effects and stop our integration at $r=3 R_{S h}$, the last stable orbit around the central black hole, where $R_{S h}=2 G M_{B H} / c^{2}$ is the Schwarzschild radius, $M_{B H}$ is the black hole mass, $c$ is the speed of light and $G$ is the gravitational constant.

Throughout the calculations the parameters of the cluster, and the mass of the central black hole, are not allowed to change and thus only stationary solutions are discussed.

Figure 1 presents a schematic representation of the internal structure of the bimodal flow induced by the presence of a central BH. In this case a fraction of the matter supplied by $\mathrm{SNe}$ and stellar winds is captured by the central $\mathrm{BH}$ and the rest is ejected as a wind into the ambient ISM.

\section{Main Equations}

The hydrodynamic equations for the steady-state, spherically symmetric flow that results from the energy and mass deposition within young and massive star clusters with a radius $R_{S C}$, mass $M_{S C}$ and mechanical luminosity $L_{S C}$, are (see, for example, Johnson \& Axford, 1971; Chevalier \& Clegg, 1985; Cantó et al. 2000; Silich et al. 2004):

$$
\begin{aligned}
& \frac{1}{r^{2}} \frac{\mathrm{d}}{\mathrm{d} r}\left(\rho u r^{2}\right)=q_{m}, \\
& \rho u \frac{\mathrm{d} u}{\mathrm{~d} r}=-\frac{\mathrm{d} P}{\mathrm{~d} r}-q_{m} u-\frac{G \rho\left[M(r)+M_{B H}\right]}{r^{2}}, \\
& \frac{1}{r^{2}} \frac{\mathrm{d}}{\mathrm{d} r}\left[\rho u r^{2}\left(\frac{u^{2}}{2}+\frac{\gamma}{\gamma-1} \frac{P}{\rho}\right)\right]=q_{e}-Q-\frac{G \rho u\left[M(r)+M_{B H}\right]}{r^{2}},
\end{aligned}
$$

and

$$
\begin{aligned}
& \frac{1}{r^{2}} \frac{\mathrm{d}}{\mathrm{d} r}\left(\rho u r^{2}\right)=0, \\
& \rho u \frac{\mathrm{d} u}{\mathrm{~d} r}=-\frac{\mathrm{d} P}{\mathrm{~d} r}-\frac{G \rho\left[M_{S C}+M_{B H}\right]}{r^{2}}, \\
& \frac{1}{r^{2}} \frac{\mathrm{d}}{\mathrm{d} r}\left[\rho u r^{2}\left(\frac{u^{2}}{2}+\frac{\gamma}{\gamma-1} \frac{P}{\rho}\right)\right]=-Q-\frac{G \rho u\left[M_{S C}+M_{B H}\right]}{r^{2}},
\end{aligned}
$$

for $r<R_{S C}$ and $r>R_{S C}$, respectively. $P, u$, and $\rho$ in equations (1- 6) are the thermal pressure, the velocity and the density of the thermalized matter. The mass and the energy 


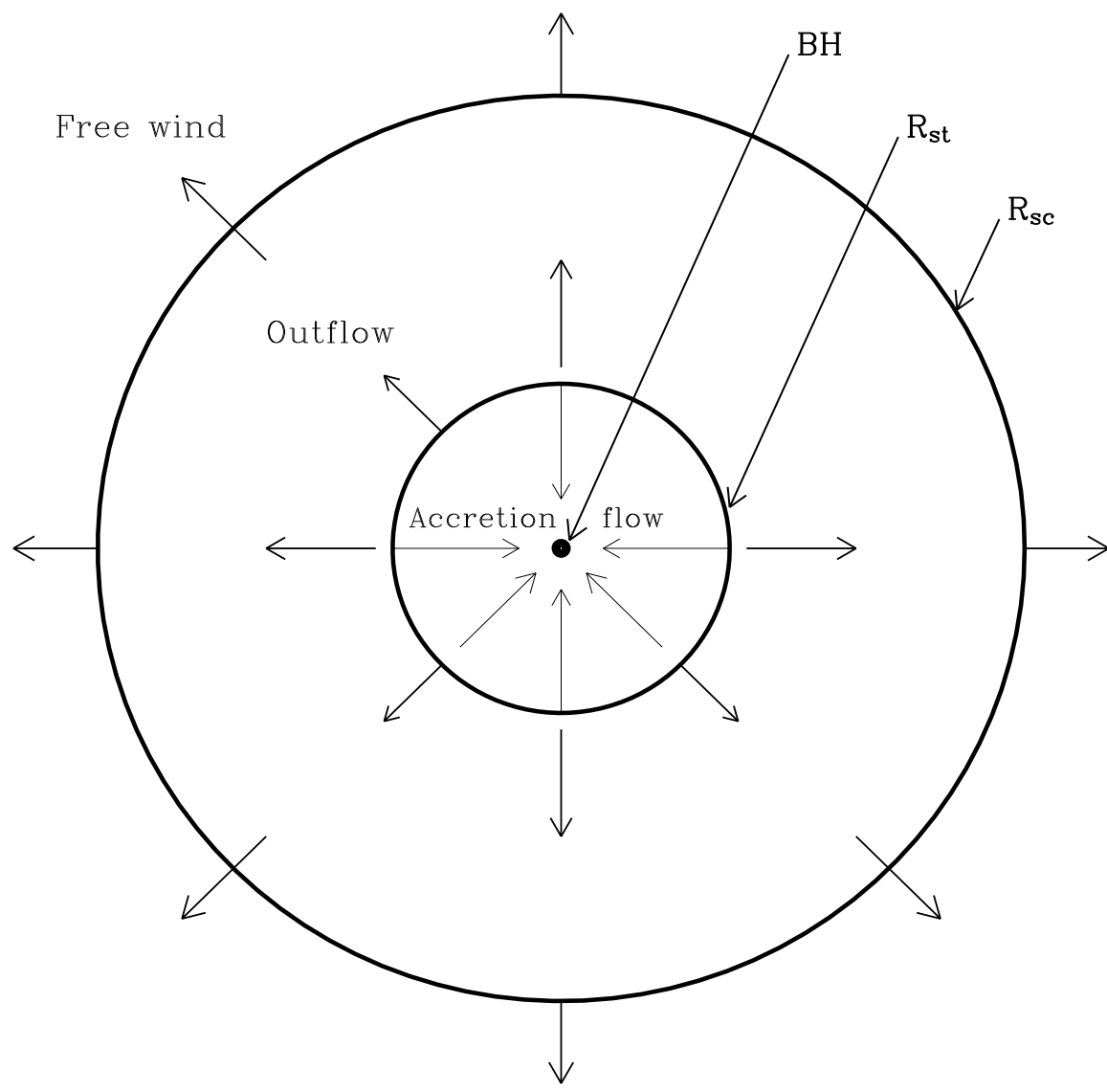

Fig. 1. - The structure of the flow that results from the thermalization of the supernova ejecta and stellar winds inside a young stellar cluster, when a massive black hole is located at the center. The radii of the internal and the external circles represent the stagnation radius $R_{s t}$ and the star cluster radius $R_{s c}$, respectively. The arrows indicate the direction of the flow. The black dot at the center marks the location of the black hole. 
deposition rates per unit volume, $q_{m}=3 \dot{M}_{S C} / 4 \pi R_{S C}^{3}$ and $q_{e}=3 L_{S C} / 4 \pi R_{S C}^{3}$, are assumed to be spatially constant inside the star cluster and equal to zero if $r>R_{S C} . Q=n_{e} n_{i} \Lambda(T, Z)$ is the cooling rate. $\Lambda(T, Z)$ is the cooling function, $T$ is the temperature and $Z$ is the metallicity of the plasma. $L_{S C}=\dot{M}_{S C} V_{A \infty}^{2} / 2$, where $V_{A \infty}$ is the adiabatic outflow terminal speed. $M(r)$ tracks the distribution of the stellar mass within the cluster, and $M_{B H}$ is the mass of the central black hole. We have taken into consideration the gravitational pull provided by the central black hole and the star cluster and neglected the self-gravity of the reinserted gaseous component.

One can easily integrate the mass conservation equation, both, inside and outside of the cluster, and rewrite equations (11-6) in the form:

$$
\begin{aligned}
& \rho=\frac{q_{m} r}{3 u}\left(1-\frac{R_{s t}^{3}}{r^{3}}\right), \\
& \frac{\mathrm{d} P}{\mathrm{~d} r}=-\rho u \frac{\mathrm{d} u}{\mathrm{~d} r}-q_{m} u-\frac{G \rho\left(M(r)+M_{B H}\right)}{r^{2}}, \\
& \frac{\mathrm{d} u}{\mathrm{~d} r}=\frac{1}{\rho} \frac{(\gamma-1)\left(q_{e}-Q\right)+q_{m}\left[\frac{\gamma+1}{2} u^{2}-\frac{2}{3}\left(1-\frac{R_{s t}^{3}}{r^{3}}\right)\left(c_{s}^{2}-V_{e s c}^{2}(r) / 4\right)\right]}{c_{s}^{2}-u^{2}},
\end{aligned}
$$

within the cluster volume, $r \leq R_{S C}$, and

$$
\begin{aligned}
& \rho=\frac{\dot{M}_{S C}}{4 \pi u r^{2}}, \\
& \frac{\mathrm{d} P}{\mathrm{~d} r}=-\frac{\dot{M}_{S C}}{4 \pi r^{2}} \frac{\mathrm{d} u}{\mathrm{~d} r}-\frac{G \dot{M}_{S C}\left(M_{S C}+M_{B H}\right)}{4 \pi r^{4} u}=-\frac{\dot{M}_{S C}}{4 \pi r^{2}}\left[\frac{\mathrm{d} u}{\mathrm{~d} r}+\frac{V_{e s c}^{2}}{2 r u}\right], \\
& \frac{\mathrm{d} u}{\mathrm{~d} r}=\frac{2 u}{r} \frac{2 \pi(\gamma-1) Q r^{3} / \dot{M}_{S C}+c_{s}^{2}-V_{e s c}^{2} / 4}{u^{2}-c_{s}^{2}},
\end{aligned}
$$

in the region $r>R_{S C} . c_{s}=(\gamma P / \rho)^{1 / 2}$ is the sound speed in the hot thermalized ejecta. The escape velocity, $V_{e s c}$, is: $V_{e s c}=\left[2 G\left(M(r)+M_{B H}\right) / r\right]^{1 / 2}$ if $r \leq R_{S C}$, and $V_{e s c}=\left[2 G\left(M_{S C}+\right.\right.$ $\left.\left.M_{B H}\right) / r\right]^{1 / 2}$ if $r>R_{S C}$, respectively.

The presence of the $\mathrm{BH}$ does not affect the relation between the gas number density and the temperature at the stagnation point found in Silich et al. 2004:

$$
n_{s t}=q_{m}^{1 / 2}\left[\frac{V_{A, \infty}^{2} / 2-c_{s t}^{2} /(\gamma-1)}{\Lambda\left(Z, T_{s t}\right)}\right]^{1 / 2}
$$

where $V_{A, \infty}=\left(2 q_{e} / q_{m}\right)^{1 / 2}$ is the adiabatic wind terminal speed, $c_{s t}$ and $\Lambda\left(Z, T_{s t}\right)$ are the sound speed and the cooling function calculated at $r=R_{s t}$. One can prove this result by 
comparing the derivative of the expansion velocity at the stagnation point obtained from equation (9) with that obtained from equation (7), and requiring a finite derivative of density at the stagnation point. Note that Sarazin \& White (1987) obtained similar relation from the energy conservation equation in their cooling flow model.

In all calculations we use the equilibrium cooling function for optically thin plasma tabulated by Plewa (1995) and assume that the metallicity of the plasma is solar.

\section{Boundary conditions and the appropriate integral curve}

The thermalization of the mechanical energy supplied by massive stars within a young stellar cluster, causes a large thermal overpressure that drives away the injected matter in the form of a high velocity outflow - the star cluster wind. The smooth transition from a subsonic expansion of the high temperature thermalized ejecta, inside the star cluster volume, to the supersonic free wind outflow at $r>R_{S C}$, requires (see equations 9 and [12) the sonic point (the point where the outflow velocity is equal to the local speed of sound) to be located at the star cluster surface (see Cantó et al. 2000; Silich et al. 2004). Hereafter we will refer to this sonic point as the outer sonic point.

In the case of stellar clusters with a central black hole, the gravitational pull of the $\mathrm{BH}$ prevents the escape of the injected matter from the central zones of the cluster and thus shifts the stagnation point from the star cluster center to a larger radius. In this case all mass continuously deposited by the cluster inside the central zone, limited by the stagnation radius, cannot escape from the gravitational well of the central $\mathrm{BH}$ and composes the accretion flow. The presence of the central BH results also in the existence of the second sonic point, between the stagnation radius and the star cluster center. To distinguish this sonic point from that at the star cluster surface we will refer to it as the inner sonic point.

Thus the stagnation radius defines the upper limit to the accretion rate onto the central $\mathrm{BH}$ and also the fraction of mass that the cluster returns to the ambient ISM. This implies that the major problem that one has to solve in order to build a self-consistent hydrodynamic solution for the flow that results from the energy and mass deposition by a young stellar cluster with a central massive BH is reduced to the calculation of the stagnation radius.

We show below that the proper position of the stagnation point is defined by the second boundary condition which is similar to that in the case of the Bondi accretion with $\gamma=5 / 3$. Specifically, we show that the inner sonic point must be located at the star cluster center. To avoid numerical problems associated with the central singularity we will assume that the inner sonic radius coincides instead with the last stable orbit associated with the central 
black hole:

$$
R_{\text {sonic }, \text { in }}=3 R_{S h},
$$

where $R_{S h}$ is the Schwarzschild radius of the central black hole.

In order to select a proper integral curve we take a trial stagnation radius and then select $T_{s t}$ from our first boundary condition which requires the outer sonic point to be located at the star cluster surface (see Silich et al. 2004; Tenorio-Tagle et al. 2007). We calculate then the number density of the plasma at the stagnation radius from equation (13) and use these $R_{s t}, T_{s t}$ and $n_{s t}$ as initial conditions to the backward integration from $R_{s t}$ towards the star cluster center.

Figure 2 presents the results of the integration for different values of the trial stagnation radius. If the selected $R_{s t}$ is too large, the backward integration leads to a double-valued, unphysical solution, marked in Figure 2 by the dashed line. In this case the velocity turnoff point (marked by a cross symbol) coincides with the inner sonic point far from the cluster center. The turnoff point moves towards $r=0$ when the considered $R_{s t}$ is smaller. This finally leads to an integral curve (marked in Figure 2 by the solid line) that approaches the last stable orbit at $R=3 R_{s h}$ with the sound speed. For even smaller $R_{s t}$ the stagnation density is larger, the accretion velocity does not reach the sonic value at $R=3 R_{\text {sh }}$ and may even go to zero as it is shown in Figure 2 (dotted line). In order to learn how the inner boundary condition affects the solution, we have provided several runs with different values of $R_{\text {sonic,in. }}$. Figure 3 shows that the stagnation radius is a weak function of $R_{\text {sonic,in }}$ and thus the inner boundary condition does not affect the solution significantly. We select as the proper solution the integral curve which has the second sonic point located at $R_{\text {sonic,in }}=3 R_{S h}$.

Figure 4 presents the distribution of the flow variables (velocity, number density and temperature) for a particular case of a $10^{8} \mathrm{M}_{\odot}$ black hole located at the center of a young stellar cluster whose mass $M_{S C}=10^{8} \mathrm{M}_{\odot}$ and $R_{S C}=40 \mathrm{pc}$. In this case the stagnation radius, marked by the inner dotted line in panel a, is $R_{s t}=2.7 \mathrm{pc}$. At larger radii the velocity grows almost linearly to reach the sonic value at the star cluster surface. It becomes supersonic outside the cluster and soon reaches the terminal value, $V_{\infty}$, somewhat smaller than the adiabatic terminal speed value, as radiative losses deplete some energy inside the cluster and in the free wind region.

In the region between the stagnation radius and the black hole, the matter deposited by stellar winds and supernovae composes a stationary accretion flow. The absolute value of the velocity grows rapidly in this region. However the flow remains subsonic as radiative losses are not able to compensate the heating of the in-flowing plasma. This leads to a rapid increase of temperature (panel c) despite the increase in density and thus of cooling of the in-falling matter, as it approaches the star cluster center (panel b). 


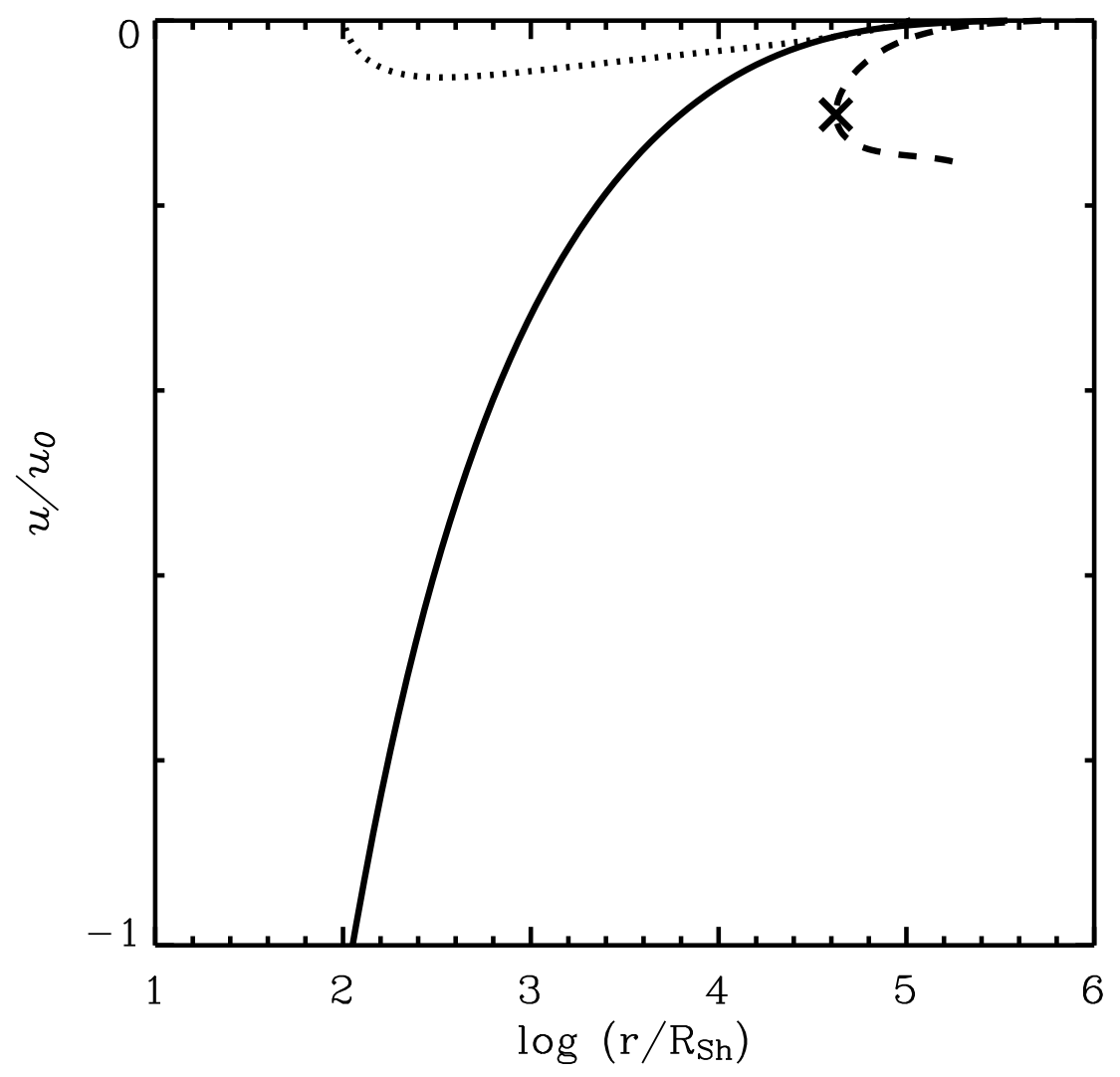

Fig. 2.- Possible integral curves. Three possible integral curves marked by dashed, solid and dotted lines correspond to different stagnation radii: $5 \mathrm{pc}, 3.3 \mathrm{pc}$ and $1 \mathrm{pc}$, respectively. The calculations assumed a star cluster mass $M_{S C}=10^{8} M_{\odot}$, a radius, $R_{S C}=100$ pc, a black hole mass, $M_{B H}=10^{8} M_{\odot}$, an adiabatic wind terminal speed, $V_{A, \infty}=1500 \mathrm{~km} \mathrm{~s}^{-1}$, and solar metallicity. The dashed line presents the unphysical double-valued solution. The solid line shows the selected solution which satisfies for both boundary conditions. The dotted line presents another unphysical branch of integral curves which tends towards positive flow velocities around the black hole. The normalization velocities are $u_{0}=10^{4} \mathrm{~km} \mathrm{~s}^{-1}$, for dashed and solid lines, and $u_{0}=10^{2} \mathrm{~km} \mathrm{~s}^{-1}$ for the dotted line, respectively. 


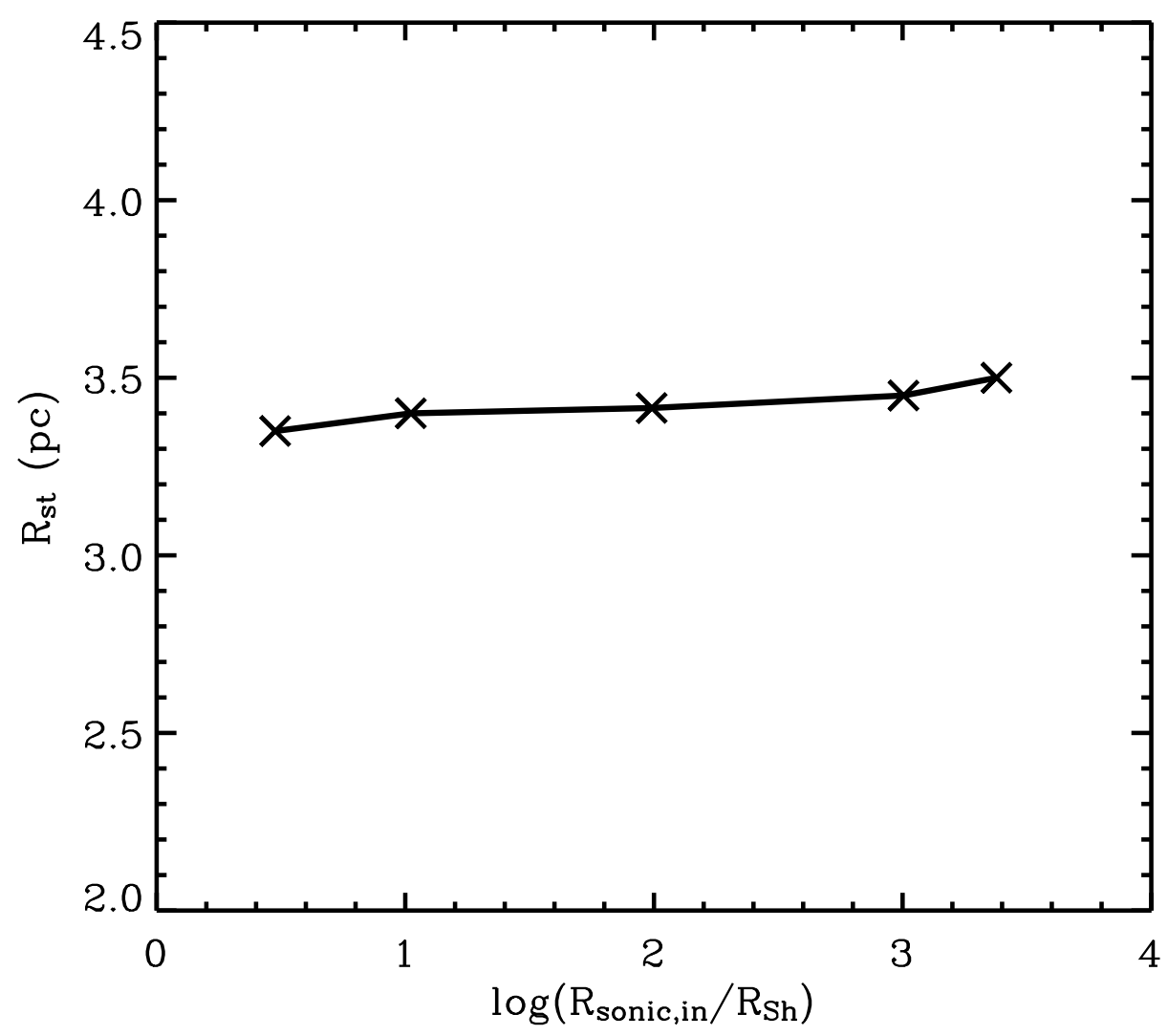

Fig. 3.- The impact of inner boundary condition on the solution. The calculations were provided for five different values of inner sonic radius: $R_{\text {sonic,in }}=$ $3 R_{S h} ; 10.5 R_{S h} ; 98 R_{S h} ; 1009 R_{S h}$ and $2385 R_{S h}$. The star cluster and black hole parameters are identical to those in Figure 2: $M_{S C}=10^{8} M_{\odot}, R_{S C}=100 \mathrm{pc}, V_{A, \infty}=1500 \mathrm{~km} \mathrm{~s}^{-1}$ and $M_{B H}=10^{8} M_{\odot}$. Cross symbols represent the results of the calculations. 

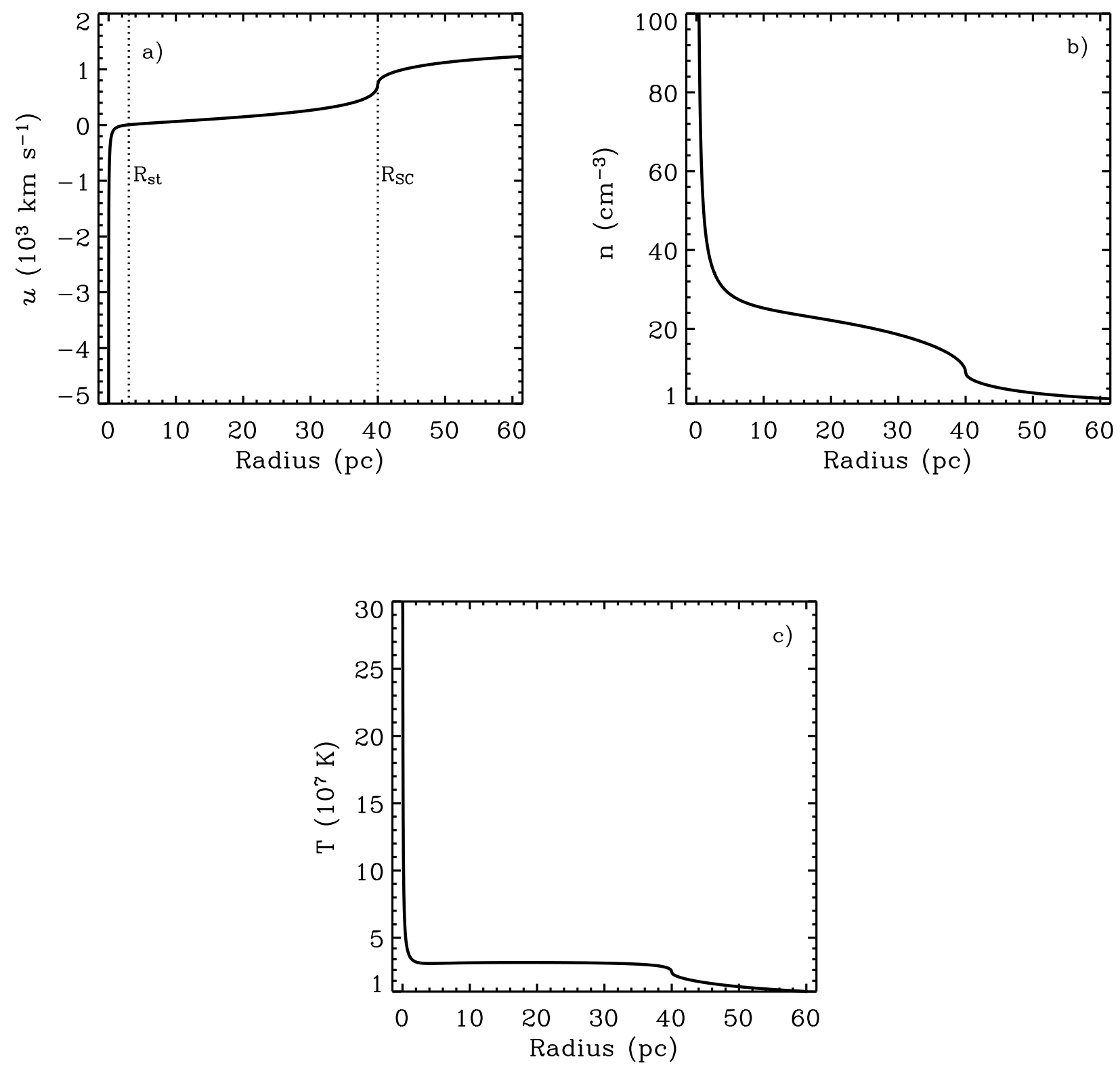

Fig. 4.- The structure of the flow in the case of a young stellar cluster with a central massive black hole. Panels a, b and c present the distribution of the flow velocity, number density and temperature, respectively. The calculations assumed a $10^{8} \mathrm{M}_{\odot}$ black hole located at the center of a young stellar cluster whose mass is $M_{S C}=10^{8} \mathrm{M}_{\odot}$ and $R_{S C}=40 \mathrm{pc}$. The adopted mechanical luminosity of the cluster is $3 \times 10^{42} \mathrm{erg} \mathrm{s}^{-1}$. This value corresponds to the average mechanical luminosity of a young stellar cluster with a Salpeter initial mass function (Leitherer et al. 1999). It was assumed that the adiabatic wind terminal speed is, $V_{A, \infty}=1500 \mathrm{~km} \mathrm{~s}^{-1}$. Vertical dotted lines in panel a mark the stagnation radius, $R_{s t}$, and the star cluster radius, $R_{S C}$, respectively. 
Figure 5 shows how the location of stagnation point depends on the mass of the black hole and that of the stellar cluster, when the radius of the cluster remains fixed. $R_{s t}$ becomes larger when the gravitational pull becomes stronger either because one considers more massive black holes or clusters.
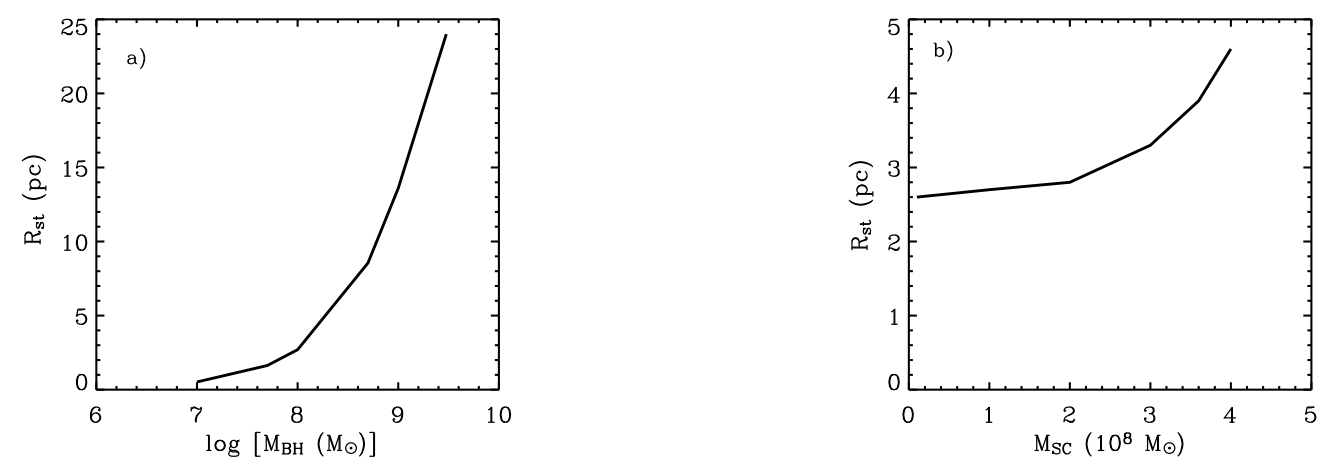

Fig. 5.- The position of the stagnation point. Panel a shows how the stagnation radius grows with the mass of the black hole for a fixed $\left(10^{8} \mathrm{M}_{\odot}\right)$ star cluster mass, whereas panel b shows the value of the stagnation radius as a function of the star cluster mass for a fixed $\left(10^{8} \mathrm{M}_{\odot}\right)$ black hole mass. In all cases the radius of the cluster is $40 \mathrm{pc}$.

\section{The threshold mechanical luminosity}

Silich et al. (2004), Tenorio-Tagle et al. (2007) and Wünsch et al. (2008) have thoroughly discussed the impact of radiative cooling on the inner structure of star cluster driven flows in the case without $\mathrm{BH}$ and without accounting for the contribution to the gravitational field imposed by the cluster. They showed that strong radiative cooling changes drastically the pressure gradient in the inner zones of compact and massive star clusters and found a critical, threshold mechanical luminosity which separates, in the $L_{S C}-R_{S C}$ parameter space, clusters with $R_{s t}=0$ pc from those evolving in the bimodal, catastrophic cooling regime. In the latter regime, strong radiative cooling promotes the displacement of the stagnation point out of the star cluster center and leads to the accumulation of the matter injected inside the stagnation volume as it becomes thermally unstable, while the outer zones of the cluster drive a stationary outflow. On the other hand, as shown above, stellar clusters with a central $\mathrm{BH}$ evolve always in a bimodal regime because the gravitational force goes to infinity when the distance to the $\mathrm{BH}$ goes to zero. This establishes the position of a stagnation point within the flow and inhibits the escape of the hot plasma from the cluster inner zones. The 
implication is thus that the two physical processes here considered: the gravitational pull from the central massive $\mathrm{BH}$ and strong radiative cooling, both promote the existence of a stagnation point within the flow and thus both compete in defining the final hydrodynamic solution.

In order to calculate the threshold energy, we choose the stagnation temperature which leads to the maximum thermal pressure at the stagnation point. This is defined by the condition that $\mathrm{d} P_{s t} / \mathrm{d} T_{s t}=0$ which, together with equation (13), yields

$$
\left(\frac{V_{A \infty}^{2}}{2}-\frac{c_{s t}^{2}}{\gamma-1}\right)\left(1-\frac{T_{s t}}{2 \Lambda} \frac{\mathrm{d} \Lambda}{\mathrm{d} T_{s t}}\right)-\frac{1}{2} \frac{c_{s t}^{2}}{\gamma-1}=0 .
$$

Then we iterate the stagnation radius and the star cluster mechanical luminosity until both boundary conditions are fulfilled, e.g. until the outer sonic point accommodates at the star cluster surface and the inner one reaches the $3 R_{S h}$ radius.

Figure 6 displays the threshold mechanical luminosity calculated for stellar clusters of different radii which contain black holes of different masses at their centers. The gravitational pull of the $\mathrm{BH}$ does not affect too much the value of the threshold luminosity in the case of large clusters. Although in all of these cases the critical luminosity is several times smaller than in the case without a BH (see Figure 6).

The gravitational pull of the BH becomes progressively more important for more compact clusters. The threshold lines turn up when the considered BH mass becomes comparable to the critical cluster mass (see Figure 6). In the case of even more compact clusters, cooling cannot compete with gravity. The position of the stagnation point is defined then by the mass of the $\mathrm{BH}$ and the stationary solution exists regardless of the cluster mass. Perhaps the only limitation here arises when $R_{s t}$ becomes larger than $R_{S C}$. This implies that the critical luminosity does not exist below $R_{S C \text {, crit }}$. The critical radii, $R_{S C, \text { crit }}$, depend on the mass of the $\mathrm{BH}$ and are marked in Figure 6 by thin vertical lines for different values of the $\mathrm{BH}$ mass.

If the cluster does not contain a $\mathrm{BH}$ and its gravitational pull is negligible, the stagnation radius is defined by the excess mechanical luminosity, $L_{S C}$, over the threshold value (Wünsch et al. 2007):

$$
\frac{R_{s t}^{3}}{R_{S C}^{3}}=1-\left(\frac{L_{c r i t}}{L_{S C}}\right)^{1 / 2} .
$$

From this it is clear that the impact of radiative cooling on the inner structure of the flow is to become progressively more important for star clusters with larger mechanical luminosities (proportional to the star cluster mass). For low mass clusters, the gravitational 


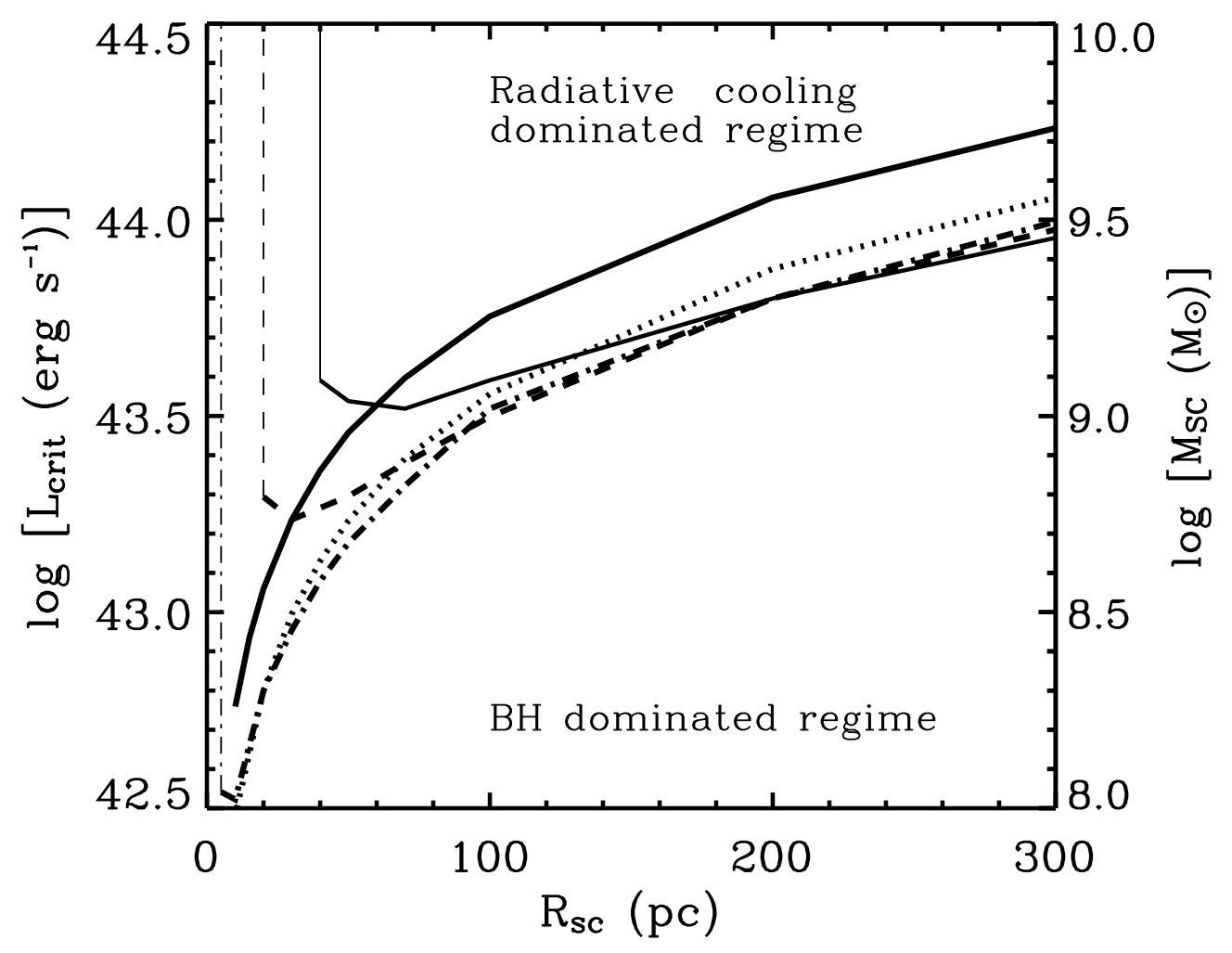

Fig. 6.- The threshold mechanical luminosity. The thick solid line marks the threshold luminosity for cases without a central black hole. The dotted, dash-dotted, dashed and thin solid lines display the threshold luminosity for clusters with $10^{7} \mathrm{M}_{\odot} 10^{8} \mathrm{M}_{\odot} 5 \times 10^{8} \mathrm{M}_{\odot}$ and $10^{9} \mathrm{M}_{\odot}$ black holes, respectively. Thin vertical lines mark critical radii, $R_{S C, \text { crit }}$. Cooling cannot compete with gravity if the star cluster radius, $R_{S C}<R_{S C \text {,crit }}$. In this parameter space gravity defines the stagnation radius regardless of the star cluster mass and the critical luminosity does not exist. It was assumed an adiabatic wind terminal speed equal to $1500 \mathrm{~km}$ $\mathrm{s}^{-1}$ and solar metallicity. 
pull of the black hole is to dominate and define the position of the stagnation point, but radiative cooling is to become an important factor when one considers more massive clusters.

We expect that above the threshold line the internal structure of the flow would present three distinct zones, as displayed in Figure 7. The outer zone would conform a quasistationary outflow. This runs from an outer stagnation radius $R_{s t, c o o l}$, set by strong radiative cooling, to meet the sound speed at the cluster surface. The central region will enclose the accretion flow onto the $\mathrm{BH}$. The outer boundary of this flow would be the stagnation radius defined by the $\mathrm{BH}\left(R_{s t, B H}\right)$. Between these two stationary flows the thermalized ejecta is thermally unstable and rapidly decays into two phases: a hot plasma whose parameters are similar to those found at the outer stagnation point, and a collection dense clouds which result from the thermally unstable plasma and are completely or partially photo-ionized by the stellar UV and BH hard radiation (Wünsch et al. 2008). These clouds can fall onto the $\mathrm{BH}$ or become gravitationally unstable and support some level of star formation inside such a cluster. Numerical calculations and a thorough discussion of this regime will be the subject of a forthcoming communication.

\section{Accretion rates and $\mathrm{BH}$ luminosities}

For clusters below the threshold line, the mass deposited by stellar winds and supernovae explosions inside $R_{s t}$ is not able to escape the stagnation volume and in the stationary regime has to fall to the center providing fuel to the central BH. Thus in the stationary regime the stagnation radius, $R_{s t}$, defines the accretion rate onto the central object:

$$
\dot{M}_{a c c}=\dot{M}_{s c}\left(\frac{R_{s t}}{R_{s c}}\right)^{3} .
$$

In this respect it is instructive to compare our theory with the classic sphericallysymmetric accretion theory (Bondi, 1952; see Frank et al. 2002 and references therein). If the polytropic index $\gamma=5 / 3$, the Bondi accretion rate is (Frank et al. 2002):

$$
\dot{M}_{B}=\pi G^{2} M_{B H}^{2} \frac{\rho_{I S M}}{c_{I S M}^{3}}
$$

where $\rho_{I S M}$ and $c_{I S M}$ are the density of the ISM and the speed of sound at infinity, respectively. We associate these quantities with Chevalier \& Clegg's central values (see Cantó et al. 2000):

$$
\rho_{c}=\frac{2}{4 \pi A} \frac{L_{s c}}{R_{s c}^{2} V_{A, \infty}^{3}}
$$




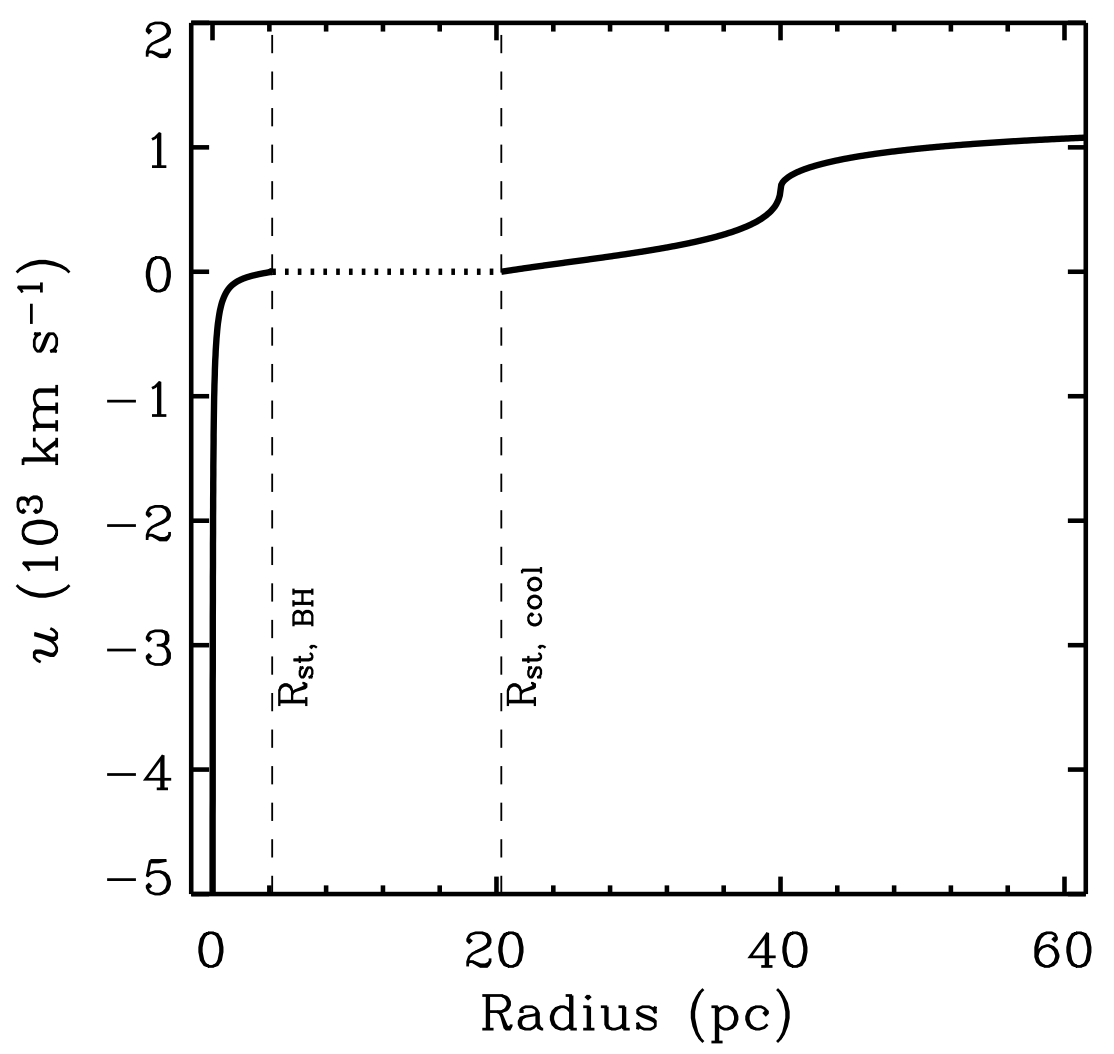

Fig. 7.- The expected velocity pattern of the flow in the case of clusters located above the threshold line. The stationary solution does not exist in this case. Positions of the inner and outer stagnation points are defined by the gravitational field of the central $\mathrm{BH}$ and strong radiative cooling, respectively. The intermediate zone is thermally unstable. 


$$
\begin{aligned}
& c_{c}=\left(\frac{\gamma-1}{2}\right)^{1 / 2} V_{A, \infty}, \\
& A=\left(\frac{\gamma-1}{\gamma+1}\right)^{1 / 2}\left(\frac{\gamma+1}{6 \gamma+2}\right)^{(3 \gamma+1) /(5 \gamma+1)} .
\end{aligned}
$$

Figure 8 compares our semi-analytic results (equation 17) with Bondi accretion rates (equation 18). The calculations were performed for a set of clusters which contain a $10^{8} \mathrm{M}_{\odot}$ $\mathrm{BH}$ at the center, all clusters have the same radius of $R_{S C}=40 \mathrm{pc}$. It was assumed that the adiabatic wind terminal speed is $V_{A \infty}=1500 \mathrm{~km} \mathrm{~s}^{-1}$ and a plasma with solar metallicity. In order to relate the mechanical luminosity of the stellar cluster to the corresponding star cluster mass we have used a relation which approximates the results of the Starburst 99 synthesis model for young stellar clusters (Leitherer et al. 1999):

$$
L_{S C}=3 \times 10^{40}\left(\frac{M_{S C}}{10^{6} \mathrm{M}_{\odot}}\right) \text { erg s}^{-1} .
$$

The star cluster mechanical luminosity has been normalized to the critical value derived in the previous section. Thus when $L_{S C} / L_{c r i t} \ll 1$, clusters evolves in the quasi-adiabatic regime, whereas when $L_{S C} / L_{c r i t} \rightarrow 1$ the star cluster parameters approach the threshold values. The semi-analytic results are marked by the cross symbols and the accretion rates predicted by the Bondi equation are shown by the solid line.

Figure 8 shows that well below the threshold line (when $L_{S C} / L_{\text {crit }} \leq 0.1$ ) Bondi's formula (equation [18) is in good agreement with our numeric results. For such clusters one can approximate the spherically-symmetric accretion rate onto a $\mathrm{BH}$ located at the center of a young massive cluster with Bondi's expression:

$$
\dot{M}_{a c c}=\pi G^{2} M_{B H}^{2} \frac{\rho_{c}}{c_{c}^{3}}
$$

where $\rho_{c}$ and $c_{c}$ are taken from equations (19) - (21). One can then use equation (23) in order to obtain an analytic expression for the stagnation radius in this $\left(L_{S C} \ll L_{c r i t}\right)$ parameter space. Indeed, the mass accretion rate is

$$
\dot{M}_{a c c}=4 \pi q_{m} R_{s t}^{3} / 3
$$

where the mass deposition rate per unit volume, $q_{m}$, is

$$
q_{m}=\frac{3}{4 \pi} \frac{\dot{M}_{s c}}{R_{s c}^{3}}=\frac{6}{4 \pi} \frac{L_{s c}}{V_{A, \infty}^{2} R_{s c}^{3}} .
$$




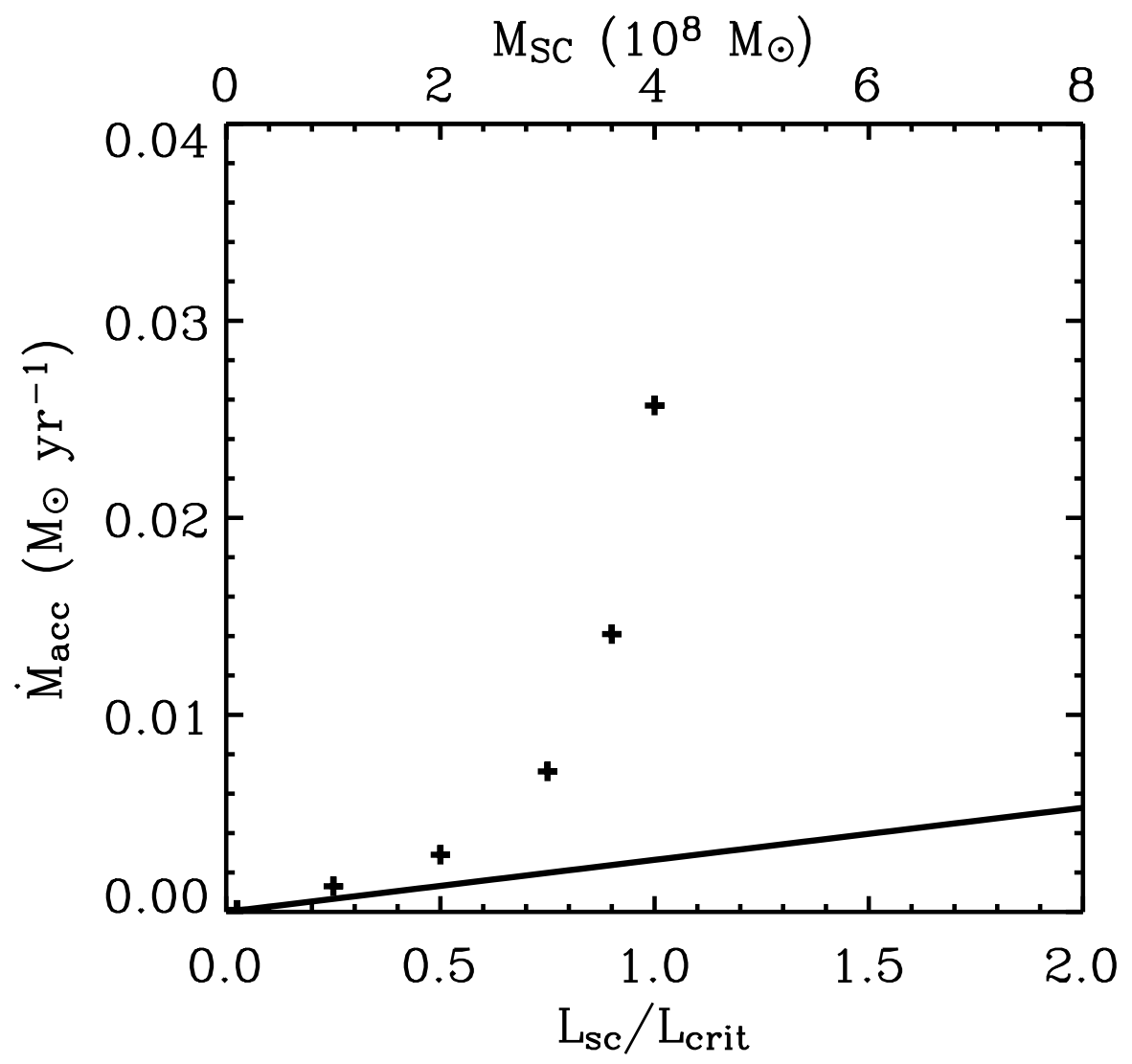

Fig. 8. - The comparison of the approximate analytic formulae with numeric accretion rates. Cross symbols present the numeric results for a $10^{8} \mathrm{M}_{\odot} \mathrm{BH}$ embedded into stellar clusters of different masses, all having radius of $R_{S C}=40 \mathrm{pc}$. Solid line displays the accretion rates calculated in the Bondi approximation with the adiabatic wind input parameters. 
Combining equations (24) and (25) with equation (23), one can obtain:

$$
R_{s t}=\left(\frac{\pi G^{2} V_{A, \infty}^{2} M_{B H}^{2} \rho_{c}}{2 L_{S C} c_{c}^{3}}\right)^{1 / 3} R_{S C} .
$$

For star clusters whose mechanical luminosities, $L_{S C}$, are comparable to the threshold value, $L_{\text {crit }}$, the calculated accretion rates exceed substantially those predicted by Bondi's equation. In this case one has to use the semi-analytic model in order to find the size of the stagnation zone and then equation (17) in order to derive the accretion rate.

Having the accretion rates and adopting the normal accretion efficiency, $\eta=0.1$, one can calculate the BH luminosity. Figure 9 compares the calculated BH luminosities with the Eddington limit, $L_{E d d}=1.3 \times 10^{38} M_{B H} / M_{\odot} \operatorname{erg~s}^{-1}$, for star clusters evolving in different hydrodynamic regimes. $L_{B H}$ grows in the case of more compact clusters. However, it remains well below the Eddington limit even when the star cluster mechanical luminosity reaches the threshold value (Figure 9, panel a). The BH luminosity can approach the Eddington limit (see Figure 9, panel b) in the case of very compact clusters whose radii are smaller than the critical values, $R_{S C \text {,crit }}$, marked in Figure 6 by vertical lines. This puts a limit for our model in the case of very compact clusters.
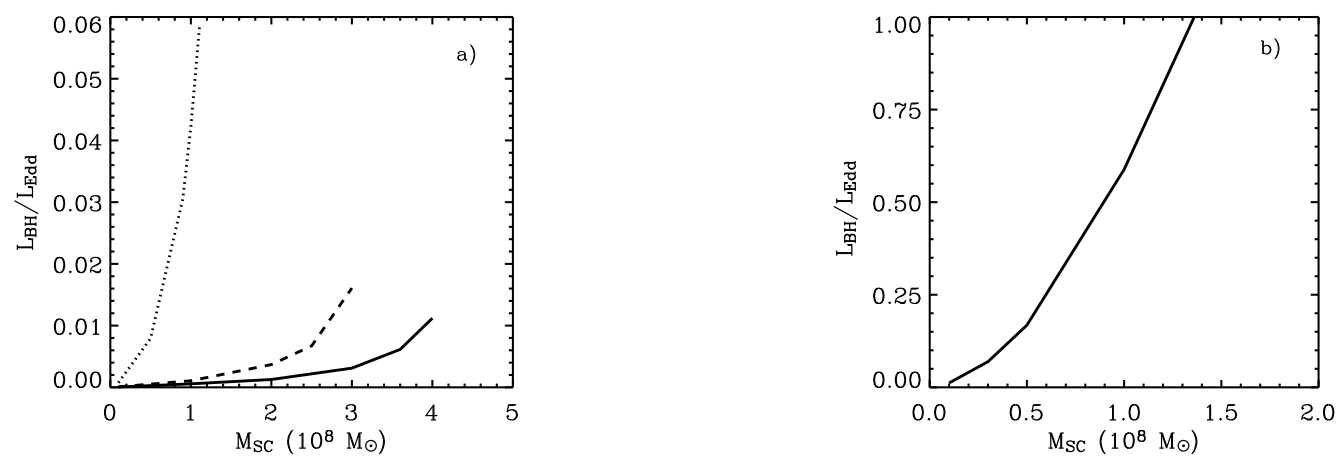

Fig. 9.- The $\mathrm{BH}$ accretion luminosity. Panel a presents the accretion luminosity of a $10^{8} \mathrm{M}_{\odot} \mathrm{BH}$ embedded into different star clusters. Solid, dashes and dotted lines display the results of the calculations for star clusters whose radii are $R_{S C}=40 \mathrm{pc}, R_{S C}=30 \mathrm{pc}$ and $R_{S C}=10 \mathrm{pc}$, respectively. The last point on every line displays the $\mathrm{BH}$ accretion luminosity when the star cluster reaches the threshold line. Panel b presents the accretion luminosity of a $10^{8} \mathrm{M}_{\odot} \mathrm{BH}$ embedded into very compact $\left(R_{S C}=3 \mathrm{pc}\right)$ star clusters, all located on the vertical dot-dashed line in Figure 6. 


\section{Conclusions}

We have developed a self-consistent, stationary solution for spherically symmetric accretion flows which are formed inside young stellar clusters with a central supermassive BH.

We have shown that the thermalization of the kinetic energy released by massive stars inside young stellar cluster results in a bimodal solution which presents an accretion of the injected matter onto a central $\mathrm{BH}$ in the inner zones of the cluster, and the ejection of the deposited matter from the outer zones of the cluster in the form of the fast superwind. We suggest that superwinds prevent the accretion of the ambient interstellar gas from the bulges and disks of their host galaxies onto the central BHs and that in such cases the BHs are fed with the matter re-inserted by massive stars in the form of numerous stellar winds and SNe explosions. The accretion rate and the BH luminosity are then defined by the central starburst but not by the gravity and the interstellar gas distribution in a host galaxy.

The hydrodynamics of the accreted and ejected matter depend on the location of the stellar cluster hosting a BH in the cluster mass - black hole mass - star cluster radius parameter space. There is a surface in this 3D parameter space which separates clusters evolving in the stationary regime from those which cannot fulfill stationary conditions. If the mechanical luminosity of the cluster exceeds the critical value, a hot plasma inside the cluster is thermally unstable. The flow is highly non-stationary and presents a complicate velocity patten with an outer stagnation point defined by strong radiative cooling and the inner one whose position is defined by the mass of the BH. The hydrodynamical structure and time evolution of the resulting flow in such cases must be calculated numerically.

Clusters whose mechanical luminosity is smaller than the critical value, compose stationary accretion flow in the central zones and form stationary outflows, the star cluster winds, in the outer zones of the cluster.

We used our model to calculate the accretion rates and the accretion luminosities of BHs at the centeres of young star forming regions. The classic, Bondi's accretion theory shows a good agreement with our semi-analytic model, but only in the case of low mass clusters located well below $\left(L_{S C} \leq 0.1 L_{c r i t}\right)$ the critical luminosity in the $L_{S C}-M_{B H}-R_{S C}$ parameter space. Thus one has to use a semi-analytic approach in order to calculate the accretion rates and $\mathrm{BH}$ luminosities in the case of more energetic clusters.

In the case of extended starbursts, the $\mathrm{BH}$ luminosities fall well below the Eddington limit. However, the accretion luminosities grow rapidly for more compact clusters and for very compact clusters can approach the Eddington luminosity, as shown in Figure 9, panel b. 
The model, here developed, is required in order to advance our knowledge regarding the relative contributions of supermassive BHs and central star bursts in composite, AGN/starburst galaxies and will be used for interpretation of observational properties of such objects in a further communication.

We thank our anonymous referee for valuable comments and suggestions. SS wishes to express his thanks to Isaac Shlosman for many useful discussions during his stay in the University of Kentucky where this study was designed. This project has been supported by CONACYT - México research grants 47534-F and 60333.

\section{REFERENCES}

Baum, S.A., O'Dea, C.P., Dallacassa, de Bruyn, A.G. \& Pedlar, A. 1993, ApJ, 419, 553

Böker, T., Laine, S., van der Marel, R. P., Sarzi, M., Rix, H.-W., Ho, L. C., \& Shields, J. S. 2002, AJ, 123, 1389

Bondi, H. 1952, MNRAS, 112, 195

Cantó, J., Raga, A.C. \& Rodríguez, L.F. 2000, ApJ, 536, 896

Chevalier, R. A. \& Clegg, A. W., 1985, Nature, 317, 44

Collin, S. \& Zahn, J.-P. 1999, A\&A, 344, 433

Côté P. et al. 2006, ApJS, 165, 57

González Delgado, R.M., Heckman, T., Leitherer, C., Meurer, G., Krolik, J., Wilson, A.S., Kinney, A. \& Koratkar, A. 1998, ApJ, 505, 174

Goodman, J. 2003, MNRAS, 339, 937

Ferrarese, L., Côté P., Bontá, E. D. et al. 2006, ApJ, 644, L21

Frank, J., King, A., \& Raine, D. 2002, Accretion Power in Astrophysics, Cambridge, Cambridge University Press, 384p.

Heckman, T., González Delgado, R.M., Leitherer, C., Meurer, G., Krolik, J., Wilson, A.S., Koratkar, A. \& Kinney, A. 1997, ApJ, 482, 114

Jiménez-Bailón, E., Santos-Lleó, M., Dahlem, M., Ehle, M., Mas-Hesse, J.M., Guainazzi, M., Heckman, T.M. \& Weaver, K.A. 2005, A\&A, 442, 861 
Johnson, H. E. \& Axford, W. I. 1971, ApJ, 165, 381

Leitherer, C., Schaerer, D., Goldader, J.D. et al., 1999, ApJS, 123, 3

Levenson, N.A., Weaver, K.A. \& Heckman, T.M. 2001, ApJ, 550, 230

Lípari, S.L. \& Terlevich, R.J. 2006, MNRAS, 368, 1001

Nulsen, P.E.J. \& Fabian, A.C. 2000, MNRAS, 311, 346

Plewa, T. 1995, MNRAS, 275, 143

Rodríguez Espinosa, J.M., Rudy, R.J. \& Jones, B. 1987, ApJ, 312, 555

Rupke, D.S., Veilleux, S. \& Sanders, D.B. 2005, ApJ, 632, 751

Sarazin, C. L. \& White, R. E. III, 1987, ApJ, 320, 32

Shlosman, I. \& Begelman, M. C. 1989, ApJ, 341, 685

Silich, S., Tenorio-Tagle, G., Rodríguez-González, A. 2004, ApJ, 610, 226

Seth, A., Agüeros, M, Lee, D. \& Basu-Zych, A. 2007, astro-ph/0801.0439

Tan, J.C. \& Blackman, E.G. 2005, MNRAS, 362, 983

Tenorio-Tagle, G., Wünsch, R., Silich, S. \& Palouš, J. 2007, ApJ, 658, 1196

Terlevich, E., Diaz, A. I. \& Terlevich, R. 1990, MNRAS, 242, 271

Walcher, C. J., Böker, T., Charlot, S., Ho, L. C., Rix, H.-W., Rossa, J., Shields, J. C. \& van der Marel, R. P. 2006, 649, 692

Wünsch, R., Silich, S. Palouš, J. \& Tenorio-Tagle, G. 2007, A\&A, 471, 579

Wünsch, R., Tenorio-Tagle, G., Palouš, J. \& Silich, S. 2008, ApJ, 684 (accepted) 